\title{
Subtraction CT angiography for the detection of intracranial aneurysms: A meta-analysis
}

\author{
TIAN-YING FENG ${ }^{1}$, XUE-FENG HAN ${ }^{2}$, RUI LANG ${ }^{1}$, FEI WANG ${ }^{3}$ and QIONG WU ${ }^{4}$ \\ Departments of ${ }^{1}$ Ultrasound and ${ }^{2}$ Emergency, Inner Mongolia People's Hospital, Hohhot, \\ Inner Mongolia Autonomous Region 010017; Departments of ${ }^{3}$ Neurosurgery and ${ }^{4}$ Magnetic Resonance Imaging, \\ Affiliated Hospital of Inner Mongolia Medical University, Hohhot, \\ Inner Mongolia Autonomous Region 010050, P.R. China
}

Received September 25, 2014; Accepted August 10, 2015

DOI: $10.3892 /$ etm.2016.3166

\begin{abstract}
The aim of this meta-analysis was to investigate the accuracy of subtraction computed tomography angiography (CTA) for diagnosing intracranial aneurysms. A systematic literature search up to January 1, 2013 was performed in PubMed. Two independent reviewers selected 8 studies that compared subtraction CTA with digital subtraction angiography. Data from the studies were used to construct a $2 \times 2$ contingency table on a per-patient basis in $\geq 5$ diseased and 5 non-diseased patients, with additional data on a per-aneurysm basis. Overall, subtraction CTA had a pooled sensitivity of $99 \%$ [95\% confidence interval (CI), 95-100\%] and specificity of $94 \%$ (95\% CI, 86-97\%) for detecting and ruling out cerebral aneurysms, respectively, on a per-patient basis. On a per-aneurysm basis, the pooled sensitivity was $96 \%(95 \%$ CI, 90-99\%), and the specificity was $91 \%$ (95\% CI, 85-95\%). In conclusion, subtraction CTA is a highly sensitive, specific and non-invasive method for the diagnosis and evaluation of intracranial aneurysms.
\end{abstract}

\section{Introduction}

The prevalence of cerebral aneurysm in the general population is estimated to be $1-5 \%$ (1). Ruptured intracranial aneurysms are the most important cause of non-traumatic subarachnoid hemorrhage, which is a medical emergency and can result in severe disability or mortality (2). Thus, the prompt diagnosis and treatment of intracranial aneurysm are of considerable importance for the outcome of the patient.

Correspondence to: Professor Fei Wang, Department of Neurosurgery, Affiliated Hospital of Inner Mongolia Medical University, 1 Tongdao Road, Hohhot, Inner Mongolia Autonomous Region 010050, P.R. China

E-mail: wangfeihol@yeah.net

Key words: subtraction computed tomography angiography, intracranial aneurysm, meta-analysis
Conventional digital subtraction angiography (DSA) has been considered the gold standard for the detection and characterization of intracranial aneurysms due to its high spatial resolution and large field of view $(3,4)$; however, it also has several disadvantages, including the relatively high cost and the high skill level required to perform the procedure. Furthermore, DSA is an invasive procedure associated with a small but definite risk of neurological morbidity (5). There is a requirement, therefore, to find an accurate, minimally invasive imaging method that is free from these complications. Computed tomography angiography (CTA), as a relatively non-invasive imaging method, has been widely used in the screening of patients with suspected intracranial aneurysms (6). As a result of the rapid improvement in multi-detector CTA technology, the diagnostic performance of multi-detector CTA for the detection of intracranial aneurysms is now approaching that of DSA (7); however, it exhibits a disadvantage in the detection of small-sized aneurysms that are located near the skull base due to the influence of overprojecting bone structures (8).

As CT technology has evolved and various subtraction and post-processing techniques have been developed, subtraction CTA, allowing bone-free visualization of aneurysms, has become possible for the diagnosis of intracranial aneurysms. There have been several reports on the potential usefulness of subtraction CTA in evaluating intracranial aneurysms; however, the results of these studies have been varied (6-8). The purpose of this meta-analysis was to calculate the sensitivity and specificity of subtraction CTA for the detection of cerebral aneurysms, in comparison with the reference standard of DSA.

\section{Materials and methods}

Search strategy. A systematic literature search up to January 1, 2013 was conducted in PubMed to identify any relevant studies. The search terms included 'tomography, X-ray computed' or 'computed tomography angiography', combined with 'intracranial aneurysm' or 'subarachnoid hemorrhage'. Studies that were evidently irrelevant, based on a scan of the titles and abstracts, were excluded, while the remaining articles were assessed for relevance to the topic of interest by reading the full text. Furthermore, a manual search was conducted by checking 
the references of retrieved articles to find any additional published studies. All searches were conducted independently by 2 authors, prior to the results being compared. Any questions or discrepancies were resolved through discussion and consensus.

Study selection. To be eligible for inclusion in the meta-analysis, the studies had to satisfy 8 inclusion criteria: i) The patients were clinically suspected of having an intracranial aneurysm; ii) the diagnostic index test was bone subtraction CTA; iii) the reference standard was DSA or its combination with neurosurgical findings; iv) the condition of interest was the presence or absence of an intracranial aneurysm; v) 2x2 contingency tables were reconstructed on a per-patient or per-aneurysm basis; vi) the study had no limitations with regard to specific aneurysm types or locations; vii) the study included $\geq 20$ patients, due to the increased likelihood of smaller studies suffering from selection bias; and viii) the study included $\geq 5$ patients with and 5 patients without an aneurysm, so that the study provided meaningful numbers for sensitivity and specificity.

Data extraction. The study data were independently extracted by 2 researchers, and any disagreements were resolved through discussion and consensus. The following data were collected: Surname of the first author, year of study publication, country in which the study was performed, study design, age range of the study participants, index test and reference standard. The $2 \times 2$ count data were extracted on a per-patient basis and, if reported, on a per-aneurysm basis.

Assessment of study quality. Study quality was assessed independently by 2 researchers using the Quality Assessment of Diagnostic Accuracy Studies (QUADAS) tool, which includes 14 quality items (9); disagreement was resolved by consensus. This evidence-based tool was developed to assess the quality of primary studies of diagnostic accuracy. The QUADAS item 4 was scored positive if the delay between the index and reference tests was $\leq 3$ days in all patients. For each study, a quality score was accumulated by assigning 1 point for each QUADAS item if fulfilled, 0.5 if unclear and 0 if not fulfilled. A score between 11 and 14 points was considered high quality and a score $<11$ points as low quality. A more detailed description of each item, together with a guide on how to score each item, is provided in the study by Whiting et al (9).

Statistical analysis. For all studies included in the meta-analysis, the individual sensitivities and specificities were recalculated from the $2 \times 2$ count data on a per-patient or per-aneurysm basis. Pooled summary estimates were obtained from a bivariate, mixed-effects, binary regression modeling framework. Model specification, estimation and prediction were performed with Stata software, version 11.0 (Stata Corp, College Station, TX, USA). A forest plot was generated that contained the individual study sensitivities and specificities with $95 \%$ confidence intervals (CIs) and the pooled sensitivity and specificity estimates. The areas under the receiver operating characteristic (ROC) curves (AUCs) were used to analyze the diagnostic precision.

The $I^{2}$ statistic was used to examine whether the results of studies were homogeneous (10). This statistic uses the conventional Q statistic to calculate the percentage of tool variation heterogeneity on a scale ranging from $0 \%$ (no heterogeneity) to $100 \%$ (all variance due to heterogeneity). In contrast to the Q statistic, the $I^{2}$ is less dependent on the number of studies included in a meta-analysis. $I^{2}>50 \%$ suggested notable heterogeneity. When statistical heterogeneity was detected, sensitivity analyses were also performed.

In studies assessing test accuracy, one of the primary causes of heterogeneity is the threshold effect, which arises due to the use of different cut-offs or thresholds in the analyzed studies to define a positive (or negative) test result. The Spearman correlation coefficient between the logit of sensitivity and the logit of 1-specificity was calculated using Meta-Disc version 1.4, in order to determine the threshold effect (11). A threshold effect was indicated by the presence of a strong, positive correlation $(\mathrm{P}<0.05)(11)$.

The presence of publication bias was visually assessed through the production of a Deeks' funnel plot and an asymmetry test (12). In the Stata software, linear regression of log odds ratios on the inverse root of effective sample sizes was performed as a test for funnel plot asymmetry. A P-value of $<0.10$ was considered to be representative of statistically significant publication bias, suggesting that only the small studies that reported a high accuracy for subtraction CTA had been published, whereas the small studies that reported a lower accuracy had not been published. Data were analyzed with Meta-Disc version 1.4 and Stata version 11.0 software.

\section{Results}

Literature search. The initial search strategy retrieved a total of 5,224 citations. Following the screening of the titles and abstracts, 5,209 sources were excluded. The full texts of the remaining 15 sources were evaluated. Of these, 7 sources were excluded for reasons given in Fig. 1 (13-19). The remaining 8 studies were included (20-27).

The study characteristics are shown in Table I. The quality assessment scores ranged from 10.5 to 13.5 , with a median study quality score of 12.5 . Four studies were prospective, 2 studies were retrospective, and in 2 studies this was unclear. The 8 studies included 982 patients. Optional count data on a per-aneurysm basis in addition to count data on a per-patient basis were provided by all 8 studies (Table I).

Assessment of publication bias. On a per-patient and per-aneurysm basis, the funnel plot and regression test showed no significant publication bias $(\mathrm{P}=0.30$ and $\mathrm{P}=0.53)$ (Fig. 2). This suggested that there were no smaller studies with lower diagnostic accuracies that had not been published.

Analysis of heterogeneity and pooled sensitivity and specificity on a per-patient basis. The sensitivities ranged from 86 to $100 \%$ (Table II). Concerning sensitivity, the selected studies showed moderate heterogeneity $\left(I^{2}=81.2 \%\right)$. For specificity, low heterogeneity was observed $\left(I^{2}=36.0 \%\right)$; the specificity ranged from 89 to $100 \%$. The overall pooled sensitivity was 99\% (95\% CI, 95-100\%), and the pooled specificity was 94\% (95\% CI, 86-97\%) (Fig. 3). The AUC was 0.99 (95\% CI, 0.97-0.99) (Fig. 4).

A Spearman rank correlation was performed as a further test for the threshold effect and was determined to be 0.558 
Table I. Studies included in the meta-analysis.

\begin{tabular}{|c|c|c|c|c|c|c|c|c|c|}
\hline First author (ref.) & Year & Country & Design & $\begin{array}{c}\text { No. of } \\
\text { patients }\end{array}$ & $\begin{array}{l}\text { Age, } \\
\text { years }\end{array}$ & $\begin{array}{l}\text { QUADAS } \\
\text { score }\end{array}$ & $\begin{array}{c}\text { No. of } \\
\text { detector rows }\end{array}$ & $\mathrm{RS}$ & $\begin{array}{c}\text { No. of } \\
\text { aneurysms }\end{array}$ \\
\hline Jayaraman (20) & 2004 & US & $\mathrm{P}$ & 35 & 54 & 13.5 & Multi-detector & DSA & 26 \\
\hline Yoon (21) & 2007 & Korea & $\mathrm{P}$ & 85 & 49.6 & 13.5 & 16 & DSA & 93 \\
\hline Romijn (22) & 2008 & Netherlands & NR & 108 & 56 & 12.0 & 4 & DSA & 117 \\
\hline Li (23) & 2009 & China & $\mathrm{P}$ & 76 & 48.0 & 12.5 & 64 & DSA & 76 \\
\hline Zhang (24) & 2010 & China & $\mathrm{P}$ & 61 & 52.0 & 11.5 & Dual-source & DSA & 47 \\
\hline Ramasundara (25) & 2010 & Australia & $\mathrm{R}$ & 36 & NA & 10.5 & $16 / 64$ & DSA & 34 \\
\hline Luo (26) & 2012 & China & NR & 56 & 48.0 & 12.5 & 320 & DSA & 51 \\
\hline Lu (27) & 2012 & China & $\mathrm{R}$ & 525 & 52.0 & 13.5 & Dual-source & 3D DSA & 459 \\
\hline
\end{tabular}

NR, not reported; P, prospective; R, retrospective; QUADAS, Quality Assessment of Diagnostic Accuracy Studies; DSA, digital subtraction angiography; 3D, three-dimensional; RS, reference standard. Data are presented as the mean.

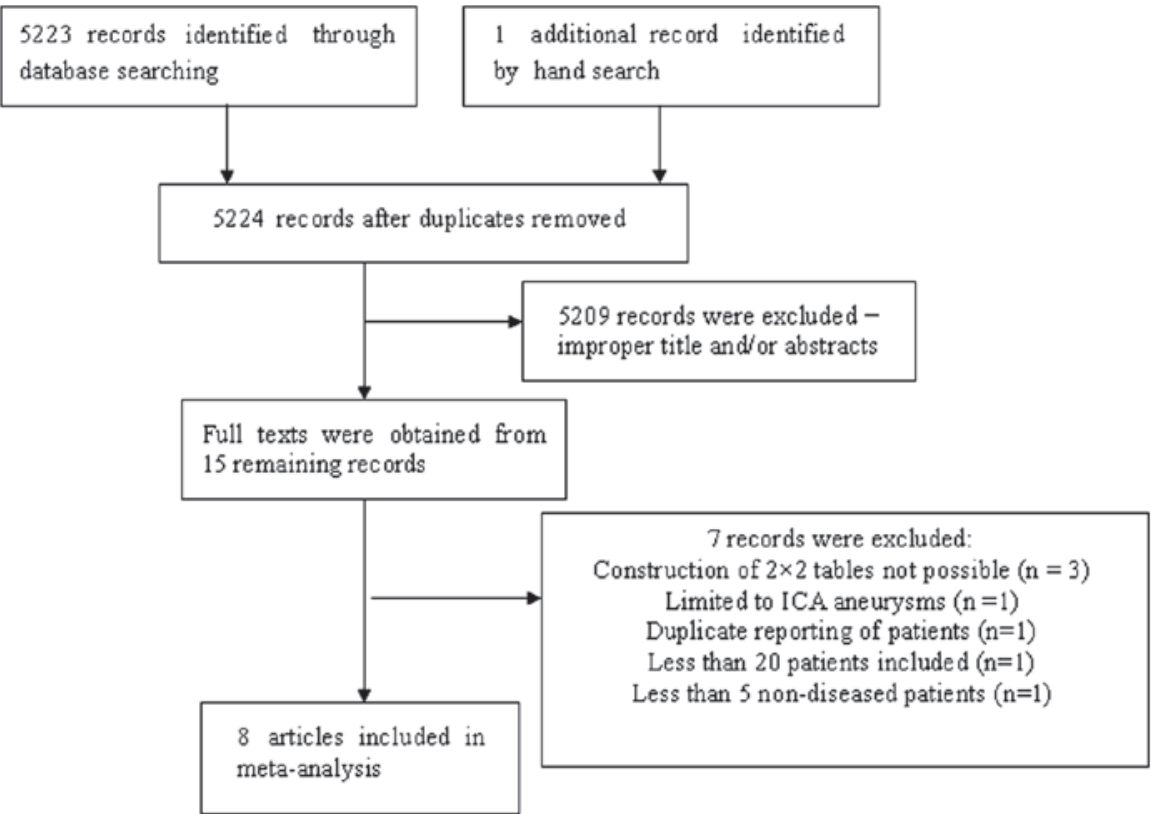

Figure 1. Flow diagram of study identification. ICA, internal carotid artery.

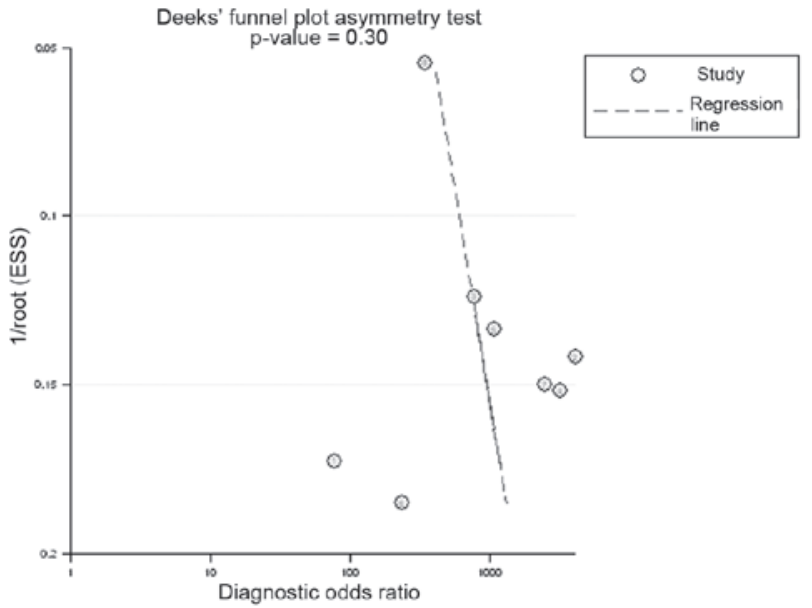

Figure 2. Deeks funnel plot asymmetry test $(\mathrm{P}=0.30)$ showing log odds ratios on inverse root of ESS for the visualization of publication bias. ESS, effective sample size.
$(\mathrm{P}=0.151)$, which indicated that there was an absence of a notable threshold effect in the accuracy estimates among the individual studies.

The results of meta-regression indicated that the year the study was published, study design, quality score and blinding method were strongly associated with sensitivity, but not with specificity (Fig. 5).

Analysis of heterogeneity and pooled sensitivity and specificity on a per-aneurysm basis. The sensitivities ranged from 77 to $100 \%$ (Table II). Concerning sensitivity, the selected studies showed moderate heterogeneity $\left(I^{2}=84.3 \%\right)$. For specificity, low heterogeneity was observed $\left(I^{2}=6.9 \%\right)$; the specificity ranged from 86 to $100 \%$. The overall pooled sensitivity was $96 \%$ (95\% CI, 90-99\%), and the pooled specificity was $91 \%$ (95\% CI, 85-95\%). The AUC was $0.96(95 \%$ CI, 0.94-0.97). 


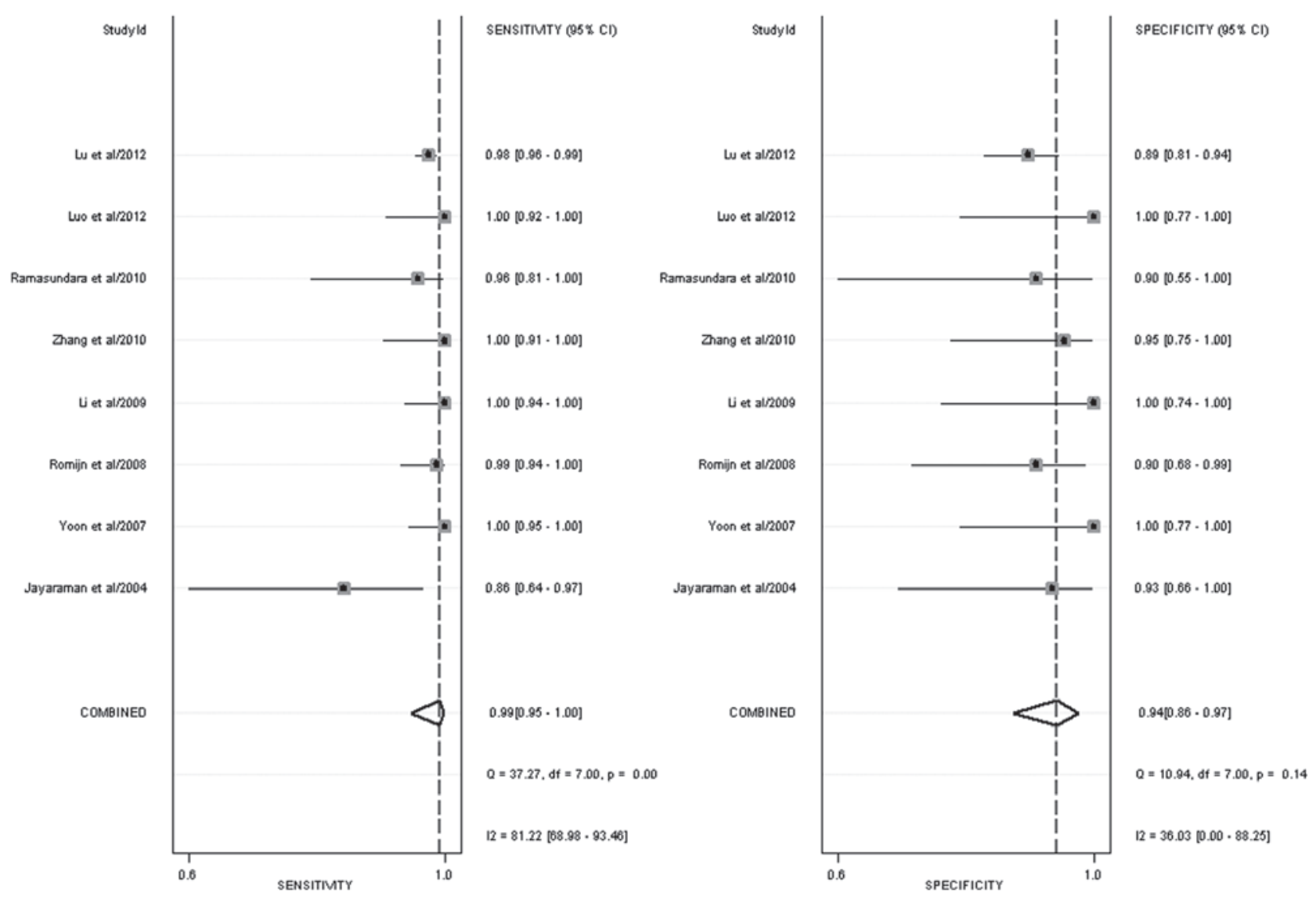

Figure 3. Forest plot shows sensitivity and specificity from individual studies and pooled estimates. Dotted squares indicate mean sensitivity or specificity for each study; horizontal lines indicate the $95 \%$ CIs of sensitivity or specificity for each study; vertical, red, dashed lines indicate pooled sensitivity or specificity for all 8 studies. CI, confidence interval.

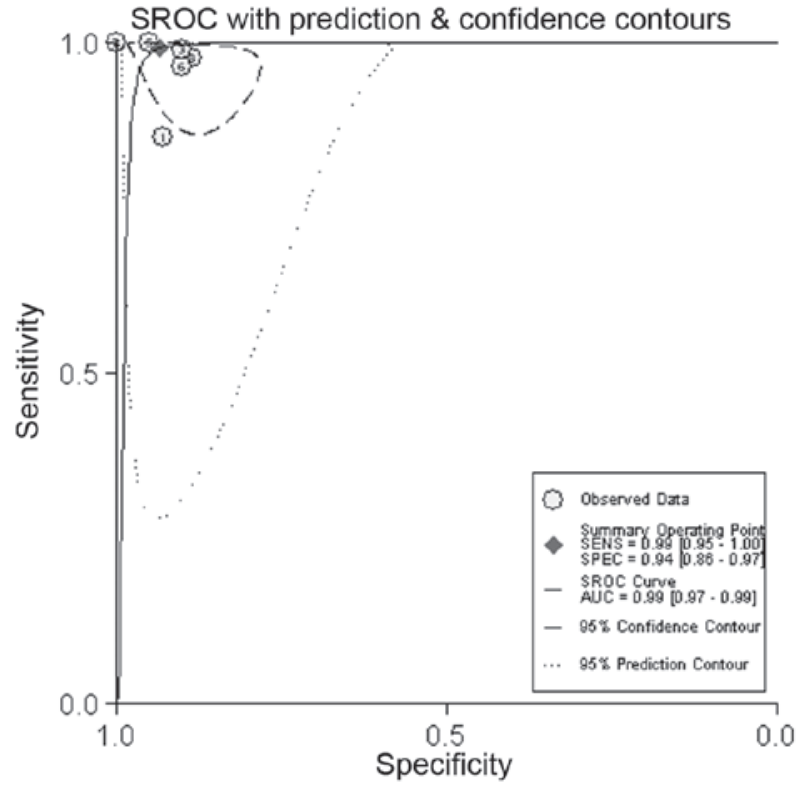

Figure 4. SROC curve with prediction and confidence contours. SROC, summary receiver operating characteristic; AUC, area under the curve; SENS, sensitivity; SPEC, specificity.

A Spearman rank correlation was performed as a further test for the threshold effect and was determined to be 0.548 $(\mathrm{P}=0.160)$, which indicated that there was an absence of a notable threshold effect in the accuracy estimates among the individual studies.

False-negative CTA results. Forty-three intracranial aneurysms were missed at subtraction CTA. The location of the false-negative aneurysm was specified for 37 aneurysms (Table III). The size of the false-negative aneurysms was also provided in 37 cases: 19 were $<3 \mathrm{~mm}, 15$ were $<5 \mathrm{~mm}$ and 3 were $5-10 \mathrm{~mm}$ in diameter. At least 22 of the missed aneurysms could be detected retrospectively.

\section{Discussion}

To the best of our knowledge, this study is the first meta-analysis of the diagnostic performance of subtraction CTA to detect cerebral aneurysms. The results show that subtraction CTA has a high diagnostic value for the detection of intracranial aneurysms. According to this meta-analysis of 8 studies, subtraction CTA has an overall sensitivity of $299 \%$ (95\% CI, 95-100\%) and a high specificity of $\sim 94 \%$ (95\% CI, 86-97\%) for diagnosing cerebral aneurysms on a per-patient basis. On a per-aneurysm basis, the diagnostic accuracy was only slightly lower.

Among the studies included in this meta-analysis, a total of 22 false-negative aneurysms at CTA could be identified retrospectively $(20,21,23,24)$. These false-negative interpretations can therefore be categorized as perceptual in nature and could have been substantially bypassed by double reading. 
Table III. Location of false-negative intracranial aneurysms.

Location

No. of aneurysms

Anterior circulation

Pericallosal artery/ophthalmic artery

Anterior communicating artery/anterior

cerebral artery

Internal carotid artery

Posterior communicating artery

Middle cerebral artery

2

5

5

9

Posterior circulation

Posterior cerebral artery

1

Posterior inferior cerebellar artery 2

Anterior superior cerebellar artery $\quad 1$

Vertebral and basilar artery

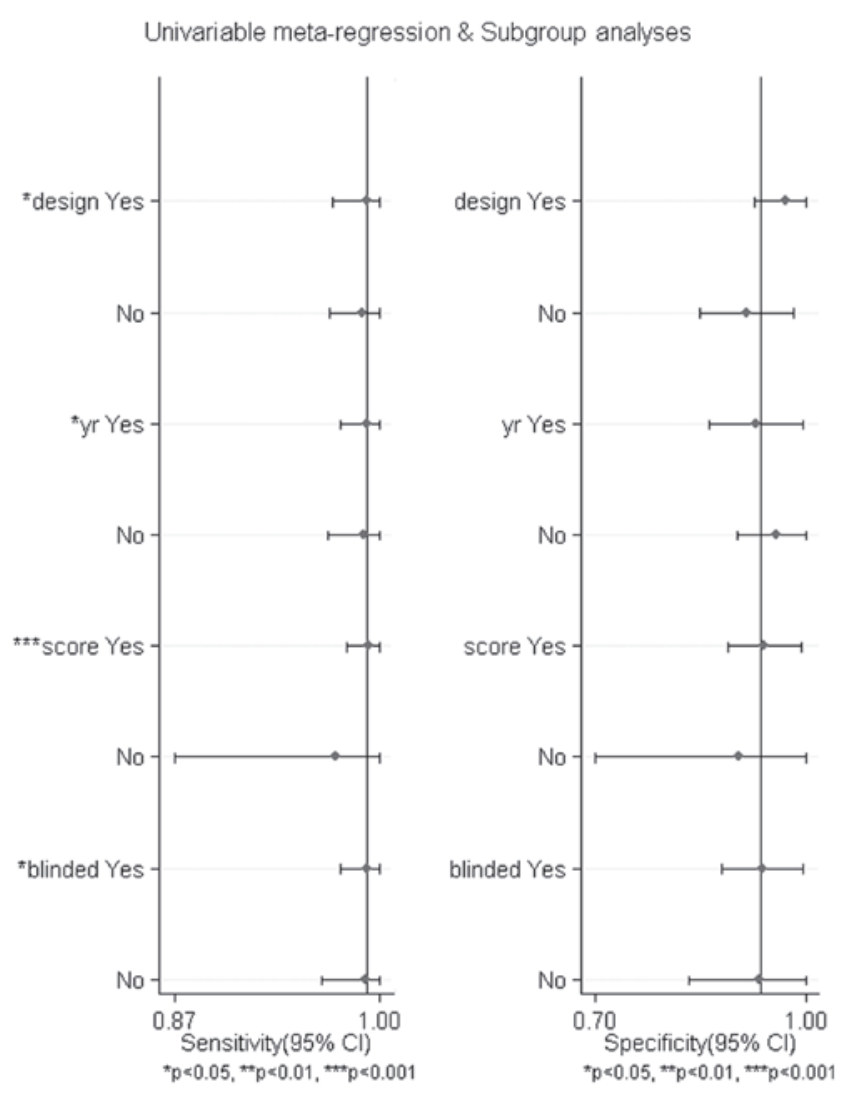

Figure 5. Univariable meta-regression and subgroup analyses on a per-patient basis. CI, confidence interval.

The $I^{2}$ statistic for sensitivity indicated the presence of notable heterogeneity, a finding that is consistent with previous meta-analyses investigating CTA and cerebral aneurysms $(7,28)$. The Spearman correlation coefficient on a per-patient basis was $0.558(\mathrm{P}=0.151)$, which suggested the absence of a significant threshold effect. To determine whether other sources of heterogeneity existed, in addition to the threshold effect, a subgroup analysis was conducted to identify factors affecting heterogeneity. The results of the meta-regression showed that the year the study was published, 
study design, quality score and blinding method had a strong association with sensitivity.

Non-subtraction multi-detector CTA has a relatively high sensitivity and specificity for the detection of cerebral aneurysms (6-8); however, the detection of aneurysms adjacent to bone remains a challenging issue due to overlying bone structures. To potentially circumvent this limitation, a number of bone removal techniques, including subtraction and manual or automated bone editing, have been developed; however, these methods are associated with several disadvantages, such as the complexity of use, dependence on the user and the high dose of radiation. Manual bone editing in CTA is a time-consuming and user-dependent process that relies on a knowledge of vascular anatomy $(13,14)$. Matched mask bone elimination (MMBE) is a relatively new technique that enables bone removal in an automatic and user-independent way $(18,22)$. In CTA-MMBE, a second, non-enhanced scan is necessary for the identification of bony structures that could be masked in the CTA scan. The two consecutive volumetric scans expose the patient to more radiation, although low-dose CT techniques are used in the non-enhanced CT. Dual-energy CTA is an immediate automatic bone removal CTA technique that offers the advantage that images from a single CT acquisition can be used to remove bone structures. This technique enables simultaneous dual-energy image acquisition in the same phase of contrast enhancement, which reduces the radiation dose. The limitation of dual-energy CT is that it is not widely available and it requires more expensive hardware (24).

A number of factors should be taken into consideration in the interpretation of the present results. First, homogeneity tests revealed moderate heterogeneity in the sensitivity of the selected studies. The potential sources of variability among the studies were variations in the quality scores, the year that the study was published, the study design and the blinding method used. Secondly, 3 studies were excluded due to the data not enabling the reconstruction of the required $2 \times 2$ count tables. It is also possible that the search of the literature did not identify all the eligible studies, but the random omission of studies is less likely to have caused a systematic bias. Although no significant publication bias was suggested by the funnel plot and regression test, unnoticed publication bias may still have been present. Thirdly, the included studies were limited to populations with a high disease prevalence. The extrapolation of the results of the meta-analysis to screening populations with a disease prevalence that is inherently lower could introduce bias. Finally, the number of studies included in this meta-analysis was relatively small; however, in a previous systematic review (29) investigating the characteristics of meta-analyses and their included studies in the Cochrane Database, it was revealed that, irrespective of the medical field, relatively few studies are typically eligible for meta-analysis for all outcomes and interventions covered by the Cochrane reviews. Furthermore, the methodological quality of the studies included in a meta-analysis has a greater impact on the estimated effects than the numbers of included studies $(30,31)$. QUADAS assessment revealed a high overall quality of the studies included in the present meta-analysis. In conclusion, the results of this study show that subtraction CTA is a highly sensitive, specific and non-invasive imaging method for the diagnosis and evaluation of intracranial aneurysms.

\section{References}

1. Wiebers DO, Whisnant JP, Huston J III, Meissner I, Brown RD Jr, Piepgras DG, Forbes GS, Thielen K, Nichols D, O'Fallon WM, et al; International Study of Unruptured Intracranial Aneurysms Investigators: Unruptured intracranial aneurysms: Natural history, clinical outcome and risks of surgical and endovascular treatment. Lancet 362: 103-110, 2003.

2. Linn FH, Rinkel GJ, Algra A and van Gijn J: Incidence of subarachnoid hemorrhage: Role of region, year and rate of computed tomography: A meta-analysis. Stroke 27: 625-629, 1996.

3. Han X, Zhan Y and Chen J: Comparative study of multi-slice CT angiography with digital subtraction angiography in the blood supply of meningiomas. Exp Ther Med 3: 31-36, 2012.

4. Brisman JL, Song JK and Newell DW: Cerebral aneurysms. N Engl J Med 355: 928-939, 2006.

5. Kaufmann TJ, Huston J III, Mandrekar JN, Schleck CD, Thielen KR and Kallmes DF: Complications of diagnostic cerebral angiography: Evaluation of 19,826 consecutive patients. Radiology 243: 812-819, 2007.

6. Chappell ET, Moure FC and Good MC: Comparison of computed tomographic angiography with digital subtraction angiography in the diagnosis of cerebral aneurysms: A meta-analysis. Neurosurgery 52: 624-631, 2003.

7. Westerlaan HE, van Dijk JM, Jansen-van der Weide MC, de Groot JC, Groen RJ, Mooij JJ and Oudkerk M: Intracranial aneurysms in patients with subarachnoid hemorrhage: CT angiography as a primary examination tool for diagnosis-systematic review and meta-analysis. Radiology 258: 134-145, 2011.

8. Donmez H, Serifov E, Kahriman G, Mavili E, Durak AC and Menkü A: Comparison of 16-row multislice CT angiography with conventional angiography for detection and evaluation of intracranial aneurysms. Eur J Radiol 80: 455-461, 2011.

9. Whiting P, Rutjes AW, Reitsma JB, Bossuyt PM and Kleijnen J: The development of QUADAS: A tool for the quality assessment of studies of diagnostic accuracy included in systematic reviews. BMC Med Res Methodol 3: 25, 2003.

10. Higgins JP, Thompson SG, Deeks JJ and Altman DG: Measuring inconsistency in meta-analyses. BMJ 327: 557-560, 2003.

11. Zamora J, Abraira V, Muriel A, Khan K and Coomarasamy A: Meta-DiSc: A software for meta-analysis of test accuracy data. BMC Med Res Methodol 6: 31, 2006.

12. Deeks JJ, Macaskill P and Irwig L: The performance of tests of publication bias and other sample size effects in systematic reviews of diagnostic test accuracy was assessed. J Clin Epidemiol 58: 882-893, 2005.

13. Schwartz RB, Tice HM, Hooten SM, Hsu L and Stieg PE: Evaluation of cerebral aneurysms with helical CT: Correlation with conventional angiography and MR angiography. Radiology 192: 717-722, 1994.

14. Imakita S, Onishi Y, Hashimoto T, Motosugi S, Kuribayashi S, Takamiya M,Hashimoto N, Yamaguchi T and Sawada T: Subtraction CT angiography with controlled-orbit helical scanning for detection of intracranial aneurysms. AJNR Am J Neuroradiol 19: 291-295, 1998.

15. Tomandl BF, Hammen T, Klotz E, Ditt H, Stermper B and Lell M: Bone-subtraction CT angiography for the evaluation of intracranial aneurysms. AJNR Am J Neuroradiol 27: 55-59, 2006.

16. Sakamoto S, Kiura Y, Shibukawa M, Ohba S, Arita K and Kurisu K: Subtracted 3D CT angiography for evaluation of internal carotid artery aneurysms: Comparison with conventional digital subtraction angiography. AJNR Am J Neuroradiol 27: 1332-1337, 2006.

17. Zhang LJ, Wu SY, Niu JB, Zhang ZL, Wang HZ, Zhao YE, Chai X, Zhou CS and Lu GM: Dual-energy CT angiography in the evaluation of intracranial aneurysms: Image quality, radiation dose and comparison with 3D rotational digital subtraction angiography. AJR Am J Roentgenol 194: 23-30, 2010.

18. Venema HW, Hulsmans FJ and den Heeten GJ: CT angiography of the circle of Willis and intracranial internal carotid arteries: Maximum intensity projection with matched mask bone elimination-feasibility study. Radiology 218: 893-898, 2001.

19. Hwang SB, Kwak HS, Han YM and Chung GH: Detection of intracranial aneurysms using three-dimensional multidetector-row CT angiography: Is bone subtraction necessary? Eur J Radiol 79: e18-e23, 2011 . 
20. Jayaraman MV, Mayo-Smith WW, Tung GA, Haas RA, Rogg JM, Mehta NR and Doberstein CE: Detection of intracranial aneurysms: Multi-detector row CT angiography compared with DSA. Radiology 230: 510-518, 2004

21. Yoon DY, Lim KJ, Choi CS, Cho BM, Oh SM and Chang SK: Detection and characterization of intracranial aneurysms with 16-channel multidetector row CT angiography: A prospective comparison of volume-rendered images and digital subtraction angiography. AJNR Am J Neuroradiol 28: 60-67, 2007.

22. Romijn M, Gratama van Andel HA, van Walderveen MA, Sprengers ME, van Rijn JC, van Rooij WJ, Venema HW, Grimbergen CA, den Heeten GJ and Majoie CB: Diagnostic accuracy of $\mathrm{CT}$ angiography with matched mask bone elimination for detection of intracranial aneurysms: Comparison with digital subtraction angiography and 3D rotational angiography. AJNR Am J Neuroradiol 29: 134-139, 2008

23. Li Q, Lv F, Li Y, Li K, Luo T and Xie P: Subtraction CT angiography for evaluation of intracranial aneurysms: Comparison with conventional CT angiography. Eur Radiol 19: 2261-2267, 2009.

24. Zhang LJ, Wu SY, Poon CS, Zhao YE, Chai X, Zhou CS and Lu GM: Automatic bone removal dual-energy CT angiography for the evaluation of intracranial aneurysms. J Comput Assist Tomogr 34: 816-824, 2010

25. Ramasundara S, Mitchell PJ and Dowling RJ: Bone subtraction CT angiography for the detection of intracranial aneurysms. J Med Imaging Radiat Oncol 54: 526-533, 2010.
26. Luo Z, Wang D, Sun X, Zhang T, Liu F, Dong D, Chan NK and Shen B: Comparison of the accuracy of subtraction CT angiography performed on 320-detector row volume CT with conventional CT angiography for diagnosis of intracranial aneurysms. Eur J Radiol 81: 118-122, 2012.

27. Lu L, Zhang LJ, Poon CS, Wu SY, Zhou CS, Luo S, Wang M and Lu GM: Digital subtraction CT angiography for detection of intracranial aneurysms: Comparison with three-dimensional digital subtraction angiography. Radiology 262: 605-612, 2012.

28. Menke J, Larsen J and Kallenberg K: Diagnosing cerebral aneurysms by computed tomographic angiography: Meta-analysis. Ann Neurol 69: 646-654, 2011.

29. Davey J, Turner RM, Clarke MJ and Higgins JP: Characteristics of meta-analyses and their component studies in the Cochrane Database of Systematic Reviews: A cross-sectional, descriptive analysis. BMC Med Res Methodol 11: 160, 2011.

30. Gluud LL, Thorlund K, Gluud C, Woods L, Harris R and Sterne JA: Correction: Reported methodologic quality and discrepancies between large and small randomized trials in meta-analyses. Ann Intern Med 149: 219, 2008.

31. Zheng Z, Li X, Li Z and Ma X: Artificial and bioartificial liver support systems for acute and acute-on-chronic hepatic failure: A meta-analysis and meta-regression. Exp Ther Med 6: 929-936, 2013. 